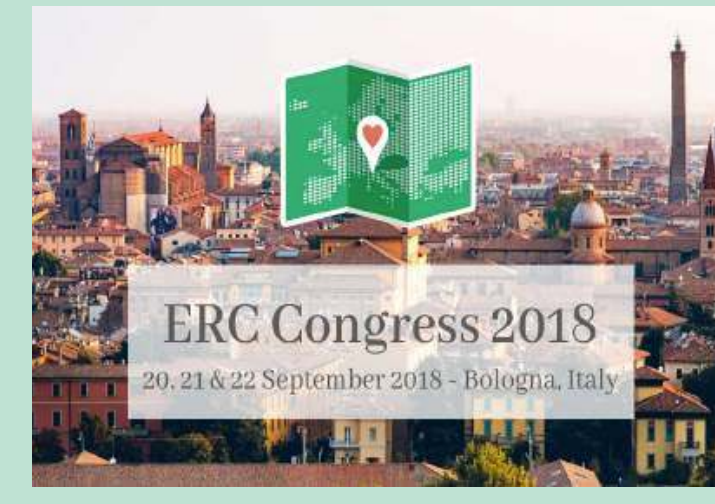

\title{
In-Hospital Extracorporeal Cardiopulmonary Resuscitation (ECPR) after prolonged Out-of-Hospital Cardiac Arrest (OHCA). A case report
}

Antonella Vezzani, Tullio Manca, Andrea Ramelli, Bruno Borrello, Andrea, Agostinelli, Andrea Moscatelli,

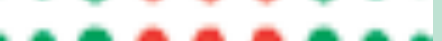

* Gaetano Gargiulo, Giorgia Paoli, Tiziano Gherli, Francesco Nicolini.

Cardiac Surgery Department of the University Hospital of Parma, ITALY; *Cardiac Surgery Department Ospedale

We present a case of a 15-year-old boy who developed pulseless electrical activity (PEA) in the school gym, after 6-minutes ride. He received effective bystander CPR performed by his teacher. Subsequent advanced CPR could not achieve a sustained return of spontaneous circulation (ROSC) and refractory cardiac arrest with PEA persisted (Fig 1). On hospital arrival US-guided percutaneous peripheral VA-ECMO was established during active CPR after $95 \mathrm{~min}$ of cardiac arrest (time from OHCA to door: $75 \mathrm{~min}$; time door to VA-ECMO: 20 min).

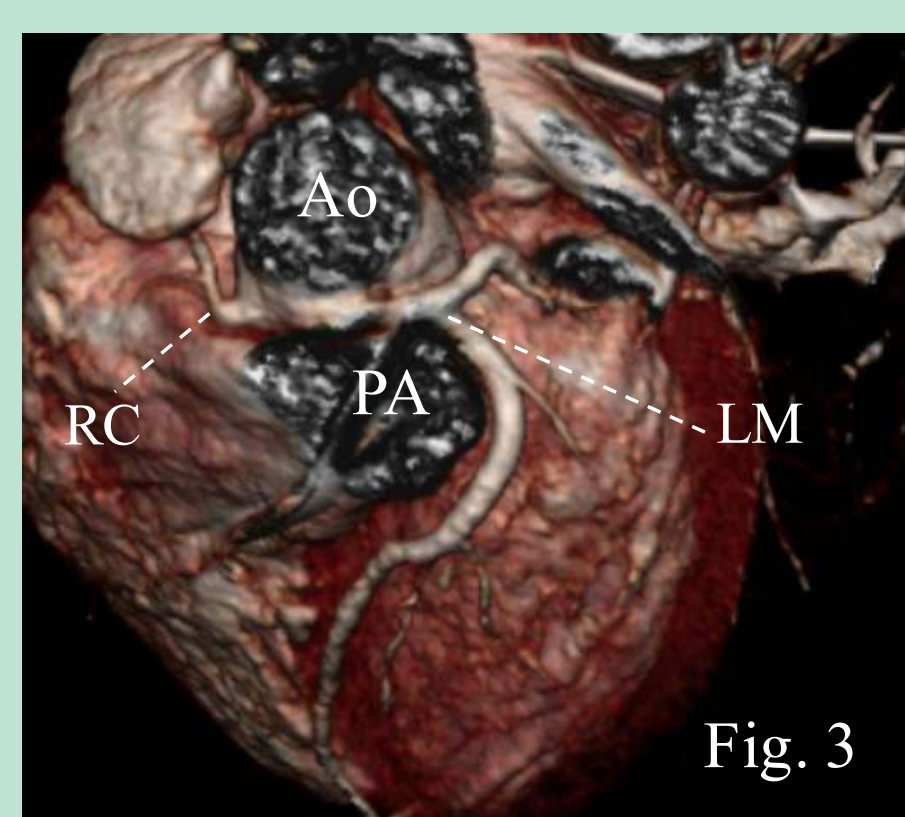

To ensure perfusion of the lower limb we performed a US-guided percutaneous cannulation of the distal femoral artery (Fig.2).

After starting ECLS an adequate systemic circulation was restored and sustained ROSC was obtained. Despite the recovery of organ perfusion, the patient showed multiple organ failure: coma, pulmonary edema, acute renal failure, metabolic acidosis, and troponin increase. Echocardiography showed severe left ventricle systolic dysfunction with
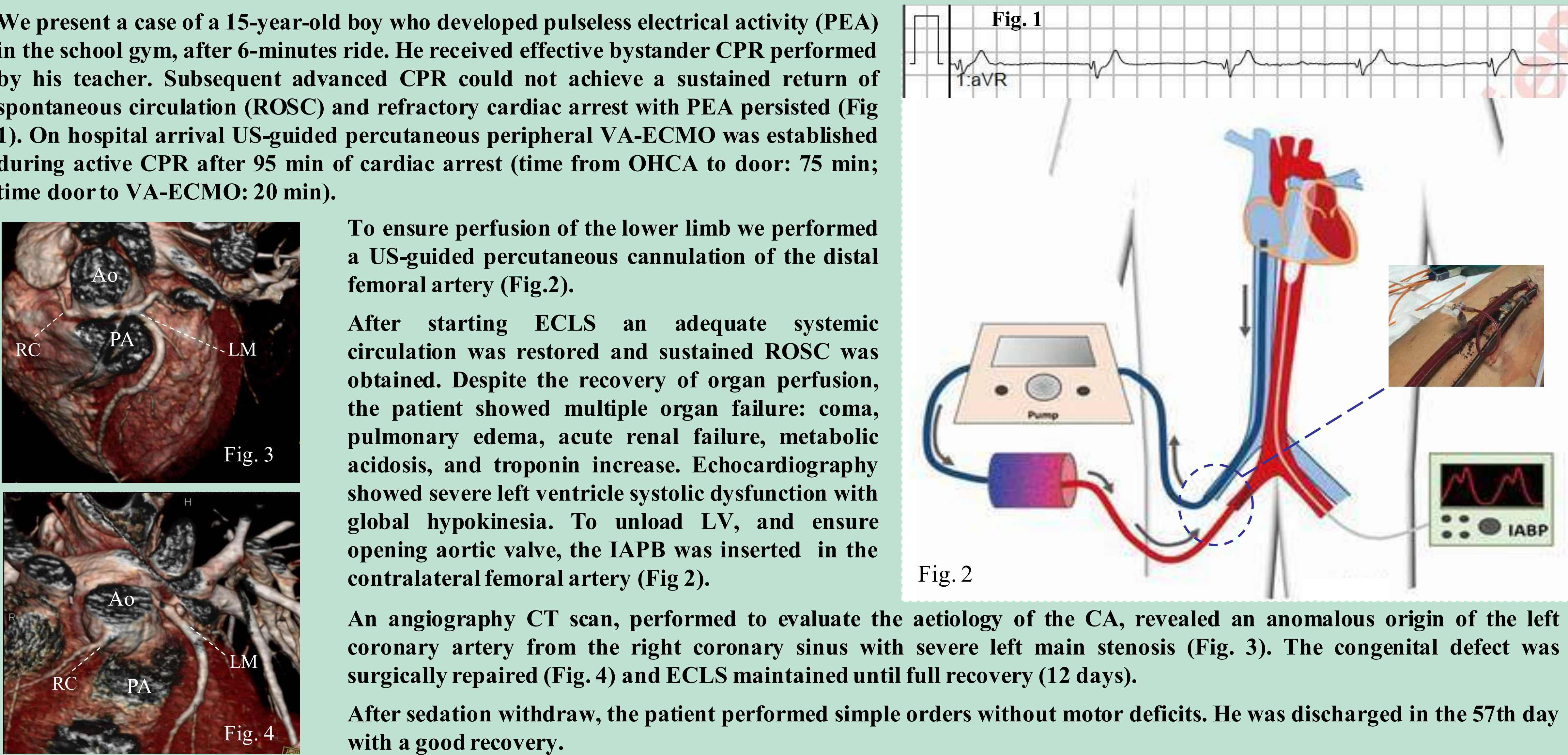
opening aortic valve, the IAPB was inserted in the contralateral femoral artery (Fig 2).

Fig. 2

An angiography CT scan, performed to evaluate the aetiology of the CA, revealed an anomalous origin of the left coronary artery from the right coronary sinus with severe left main stenosis (Fig. 3). The congenital defect was surgically repaired (Fig. 4) and ECLS maintained until full recovery (12 days).

After sedation withdraw, the patient performed simple orders without motor deficits. He was discharged in the 57th day with a good recovery.

Conclusion: In refractory cardiac arrest, ECPR has several advantages, including successful ROSC, maintaining organ perfusion during recovery of native cardiac output, earn time to perform diagnostic tests and apply surgical and medical treatments. 